\title{
Profetismo y Mesianismo en la teología política de Maimónides
}

Prophecy and Messianism in Maimonides political theology

\author{
Ignacio Cid \\ iecidpuc@gmail.com \\ Universidad Católica de Chile \\ Chile
}

\section{Resumen}

En el presente artículo se describen algunas de las tendencias centrales tanto de la profetología como del pensamiento mesiánico de Maimónides. A través de dos de los momentos centrales de la historia judía, se desentraña la teología política del autor como el transitar entre la recepción de la ley y su cumplimiento integral.

Palabra claves: Mesías - Ley - Profeta - Moisés.

\begin{abstract}
This article describes some of the central tendencies of Maimonides' prophecy and messianic thinking. Through two of the central moments of Jewish history, the political theology of the author is unraveled as the transit between the reception of the law and its integral fulfillment.
\end{abstract}

Keywords: Messiah - Law - Prophet - Moses. 
Contadas son las tradiciones religiosas en las que la profecía en tanto theologia revelata, juegue un papel tan relevante como en el judaísmo. Aún menos son las religiones en las que se le atribuye a la profecía una dimensión marcadamente política. Ambos elementos se conjugan de modo integral en la reflexión judía en torno al problema de la ley, el modo de inspiración profético y el ideal mesiánico.

A la luz de la mediación que ejerce el texto entre el pueblo judío y su Dios, se ha desarrollado a través de los siglos, una extensa literatura respecto a la cuestión del Mesías, el Profeta y la Ley. El triunfo del rabinismo a partir de la destrucción del templo en el año 70 d.c, inaugura un periodo de intenso intercambio intelectual respecto al papel Ley y especialmente del tiempo mesiánico.

Es precisamente esta tradición rabínica la que, a pesar de ser oficialmente clausurada con el Talmud, alcanza su punto culminante con el trabajo de recopilación y sistematización del rabino Moshé ben Maimón, conocido en los ambientes latinos como Maimónides y en los hebreos con el acrónimo de Rambam.

Maimónides nació en Córdoba el año 1135 y falleció en el Cairo en 1204. Junto a Saadia Gaon fue uno de los más destacados representantes de la filosofía judía medieval. Como ávido lector de Aristóteles, incorporó elementos de su teoría al judaísmo. Fue uno de los primeros pensadores judíos en elaborar una metafísica y se vio en la obligación de legitimar la actividad filosófica frente al estudio de la ley y la jurisprudencia. Durante su estancia en el Al-Andalus, se nutrió de un ambiente intelectual enriquecido por filósofos musulmanes como Avicenas y Al-Farabi, a los que leyó de manera cercana y atenta. Admiró de sobremanera, su capacidad de síntesis entre tradición religiosa y el legado de la filosofía peripatética.

A partir de ese hecho, en su libro Guía de perplejos, el filósofo medieval inaugura su reflexión justificando la compatibilidad entre la razón filosófica y el pensamiento religioso judío. Es importante recordar a este respecto que si "para el cristianismo, la doctrina sagrada es la teología revelada; para el judío y el musulmán, la doctrina sagrada es, al menos en lo primordial, la interpretación legal de la Ley Divina (talmud o fiqh)” (Strauss, 
2009, pág. 24). En ese sentido, la síntesis que propone el trabajo de Maimónides se hace cargo de reivindicar el pensar filosófico subordinado a los límites que le impone la revelación divina en su forma de Torá.

Lo anterior no debe conducir a la conclusión errónea de que en Maimónides lo teológico y lo filosófico son dos vías análogas conducentes a una “doble verdad”. Muy por el contrario, mientras la filosofía marca el camino de los hombres, la Torá (y la teología que a ella subyace) invita al hombre a transitar por él. La filosofía es en ese sentido para Rambam, una herramienta que ilumina los oscuros e intrincados caminos que subyacen al pensamiento teológico (Taub, 2014).

Dado este hecho, la primera sección de la Guía arranca con la metafísica. En estos pasajes, Maimónides examina algunas de las cuestiones fundamentales para el pensamiento judío, desde una perspectiva filosófica como el intelecto, la eternidad del mundo, el sentido filosófico de Dios y las tesis sobre la creación del universo. Excluye, sin embargo, la cuestión de la ley y la inspiración profética, bajo el supuesto de que ambos asuntos no le competen a la metafísica sino a la política. Esto adelanta ya un marcado componente político que adquieren los conceptos rabínicos en el pensamiento del Rambam y que culminan en su idea del mesías y la era mesiánica.

En la segunda sección del libro, Maimónides define la profecía como "una emanación de Dios, que se extiende, por mediación del intelecto agente, sobre la facultad racional, primero, y sobre la facultad imaginativa" (Maimónides, 2005: 334). A continuación, explica la necesidad de esta doble proyección de la profecía, arguyendo que, si la emanación de Dios alcanzara solo la facultad racional del profeta, éste se identificaría únicamente con "la clase de lo sabios y de la gente de especulación" (Maimónides, 2005: 334). Asimismo, si la emanación estuviera referida exclusivamente a la facultad imaginativa, "la clase constituida así sería la de los gobernantes de las ciudades y los legisladores, los adivinos y los augures que sueñan sueños verdaderos y también los que hacen milagros con artificios extraordinarios y artes ocultas sin ser sabios" (Maimónides, 2005: 338). 
Maimónides identifica así la figura del profeta, con la de un hombre "interesado sólo en el conocimiento de la deidad y en la reflexión de Sus obras y en lo que debe ser creído respecto a ello” (Maimónides, 2005: 389). De este modo afirma que, dada la perfección de su intelecto por las cosas que le han sido reveladas, el profeta tiene un conocimiento perfecto de las verdades especulativas de Dios. En ese sentido, el profeta es el maestro de los hombres. Solo él tiene acceso al contenido esotérico de la profecía, por tanto, su labor es explicarla, mediante analogías y metáforas, a una audiencia de hombres con capacidades muy disimiles para recibirla.

Además de esta profetología, Maimónides deja entrever la influencia que ejercen los falasafi o "musulmanes helenizantes" sobre su pensamiento. De acuerdo con Al-Farabi, a quien Maimónides consideraba solo inferior a Aristóteles, la autoridad política es al mismo tiempo una autoridad profética, precisamente por su adhesión a la sharia, (ley divina). La legitimidad que el gobernante puede alcanzar entonces, según Al-Farabi, está supeditada a una ley cuyo fin es orientar a los ciudadanos hacia la virtud que constituirá la ciudad ideal. De este modo, enseña que el "primer jefe" de la ciudad ideal ha de ser "profeta" e "imam" (Strauss, 2012).

La idea de "primer-jefe" sigue una trayectoria que puede ser trazada hasta el concepto de “filósofo-rey" en Platón, como único facultado para gobernar en virtud de su capacidad de acceder al Bien. En base a estas concepciones, Maimónides prefigura la idea de un profetagobernante, dejando en evidencia una teología política que se revela, en principio, como fuertemente platonizante. La corrección judía de esta tendencia proviene, sin embargo, de manos de su doctrina de la primacía del profeta sobre el filósofo. Según Rambam mientras el hombre común está limitado al conocimiento del mundo sensible, el profeta lo supera de sus facultades sobrenaturales. Así, la enseñanza de Aristóteles que concierne al mundo sublunar, es considerada correcta en todos los aspectos. Sin embargo, sus enseñanzas sobre el mundo lunar, es decir, sobre la correcta opinión de Dios y los ángeles, no es perfectamente correcta y en ese sentido, es complementada y superada por aquella que viene del profeta. De este modo, "el profeta es superior al filósofo en el punto mismo en el que el filósofo es superior a todos los no filósofos" (Lerner, 1998: 344). 
El gobierno del profeta deber estar asentado sobre la ley divina en la medida que ella tiene por fin el bienestar del cuerpo y del alma. A diferencia de la ley civil, que solo sirve para el ordenamiento recto de la ciudad, la ley que es revelada ayuda a los ciudadanos en "la adquisición de las opiniones debidas y del conocimiento auténtico por medio de la especulación” (Lerner, 1998: 344). Con esto, Maimónides parece sugerir que la política como ámbito de la vida humana debe estar sujeta exclusivamente a la ley.

La doctrina de la supremacía de la ley sobre la política se entronca perfectamente con el acento profetológico de la teología política de Maimónides, toda vez que lo identifica como mecanismo originario: "La creencia en la profecía precede a la creencia en ley, pues si no hay profeta, no hay ley" (Lerner, 1998: 232). Con esto, la Torá como ley divina que es perfecta, es revelada y entregada al pueblo judío a través de Moisés en el Sinaí, con el fin de fundar la nación perfecta. Este hecho constituye para Maimónides, la raison d'etre de la profecía.

A esta luz, subyace a la política y su búsqueda de la "ciudad ideal" cierta irrelevancia frente a la Torá. La política revela así, su condición superflua ante la grandeza de una ley que regula todos y cada uno de los aspectos de la vida humana (Strauss, 2012: 222). Ella es el hecho político por antonomasia, una ley ideal que constituye la ciudad ideal, el nomo de toda política.

Con el fin político de la ley consolidado, Rambam introduce la teoría de la superioridad de la profecía de Moisés respecto a la profecía ordinaria. Escribe:

"Después de hablar de la esencia de la profecía, que hicimos conocer en su verdadero estado, y exponer que la profecía del profeta Moisés, nuestro maestro, se distingue de la profecía de los demás, diremos que solo esta precepción ha tenido como consecuencia necesaria apelar a la ley”. (Maimónides, 2005: 342) 
La operación que aquí propone el filósofo judío, es elevar la profecía de Moisés porque solo ella ha tenido como resultado la ley. Los profetas que lo antecedieron, así como aquellos que le sucedieron, han tenido por labor anunciar, proteger y confirmar dicha ley, de modo que se han subordinado a ella. Moisés como jefe de la profecía, es el más político entre los profetas, pues es quien funda la comunidad perfecta a través de la legislación perfecta, la ley de Dios.

Con esta superioridad en la profecía de Moisés, Maimónides elabora su propia teoría en torno a la autoridad política como profeta-legislador. Nuevamente se distancia del "primerjefe" de Al-Farabi, pues si para el filósofo musulmán la ciudad ideal se funda temporalmente, mediante un acto legislativo en el que la sharia es promulgada, para Maimónides, la Torá funda de una vez y para siempre la comunidad política como nación perfecta. Precisamente el concepto de nación distingue al pueblo judío de la ciudad perfecta y cualquiera de sus realizaciones históricas. La nación hebrea supera el espacio de la polis, porque en su seno aloja la perfección que solo puede provenir de la Ley. Esta última idea, es de particular relevancia dada la situación de diáspora en la que viven los judíos de la época en las distintas ciudades de Europa, condición en la que se encuentra el propio Maimónides. Un pueblo desterrado y sin tierra, no requiere de una ciudad para vivir virtuosamente, aunque anhela volver a su tierra para observar los preceptos de Dios.

Finalmente, la profetología de Maimónides se corona con la teoría de la inmutabilidad de la ley. La Torá que recibe Moisés es absoluta y revelada para la eternidad. Su sacralidad está dada por su origen divino que, a su vez, da cuenta del plan del Creador del universo, perfecto e inamovible para la voluntad humana. Ese es el sentido de que se afirme que "ningún profeta podrá presentarse a querer cambiarla, y tampoco aparecerá un nuevo Moisés para introducir una nueva ley que la reemplace” (Davis, 1952: 52).

A esta luz, la profecía legislativa en Maimónides es la fuente de la que se desprende toda política. Ella constituye a Israel como "pueblo elegido" con "deberes excepcionales" a la frente a la humanidad. Se rechaza la idea de superioridad de Israel a través de "derechos excepcionales" realzando en su lugar, la noción de "pueblo ético" llamado a guiar a las 
naciones a cumplir los preceptos de Dios (Karmy, 2016: 70). Isaías 49:8 advierte que "Por eso te pondré como una luz para las naciones, y haré que lleves la salvación hasta el último rincón del mundo”.

La invitación a que otras naciones imiten a Israel en el seguimiento de su ley, no debe ser entendido como un acto proselitista por parte de Maimónides. El autor piensa más bien, que la profecía y el tiempo venidero están marcados por una humanidad en la que el respeto hacia la ley y el reconocimiento de Dios único se extenderán. La figura de las leyes noajidas entregadas por Dios a Noé luego de destruir y repoblar la tierra, son de extrema utilidad para Rambam. Precisamente el carácter universal, que contrasta con el particularismo de la profecía de Moisés, descansa en que las leyes de Noé incluyen al nojudío en la ley y por lo tanto en la política. Su cumplimento se produce en el contexto del mundo presente, y sin embargo, se afirma que "quien acepte las siete leyes [noájidas] y es cuidadoso observándolas es considerado un "justo de las naciones" y tendrá un lugar en el mundo por venir" (Maimónides, 1963: 230).

A partir de la ley y especialmente de las leyes noajidas, Maimónides establece un vínculo entre el mundo en el que la profecía tiene lugar y aquel en el que ella cesa en favor del tiempo mesiánico. Con ese objetivo, lee la historia en función del despliegue del monoteísmo identificando cuatro periodos: "la era de la ignorancia, la era en la que el monoteísmo comienza y tiene sus raíces en una simple comunidad, la extensión del monoteísmo, y la era del triunfo del monoteísmo: la era mesiánica” (Taub, 2012: 12).

Según su propia percepción, el maestro medieval estaría viviendo en la etapa de extensión del monoteísmo. Aunque idólatra, Rambam ve en el avance del cristianismo y el islam una extensión del monoteísmo que descubrió Abraham en la tienda de su padre Teraj. De este modo, la época que lo antecedió y donde el monoteísmo se ve alentado por la profecía está clausurada. De allí que Maimónides viva sus días bajo la expectación de un tiempo mesiánico que es inminente.

Desde el comienzo de su reflexión, el autor establece el concepto de Mesías como una figura personal e histórica, que viene a inaugurar una era que es punto de consagración 
entre la historia humana y la de Dios. El mesías tiene por misión resolver la tensión que subyace a la relación entre la universalidad de lo político y el particularismo de lo profetológico. Es por eso que su labor es para Maimónides, la de un liberador nacional que restaurará el reino de Israel:

“El Rey Mesías se presentará y restaurará el reino de David a su estado anterior y a su soberanía original. Él reconstruirá el santuario y reunirá a los dispersos de Israel. Todas las leyes antiguas serán reinstituidas en sus días”. (Maimónodes, 1963: 238)

La doctrina del mesías resulta fundamental para la teología política de este autor medieval toda vez que enfrenta a quienes abandonaron la esperanza mesiánica, producto de los excesos cristiánanos; Maimónides sostiene así que "aquel que no cree en la restauración o no espera la llegada del Mesías niega no sólo las enseñanzas de los Profetas, sino también la Ley de Moisés" (Maimónides, 1963: 238). Es posible observar en esta aseveración que, si bien el mesías viene a resolver el problema político de la profecía en un mundo idólatra donde la ley no se cumple en su totalidad, él no la supera ni la modifica. Escribe Rambam a este respecto:

“Cualquier profeta que surja, ya sea sacerdote, levita o amalequita, y diga que hay que quitar obligatoriedad a uno solo de los preceptos contenidos en el Libro de la Torá, miente y niega la profecía de Moisés, nuestro maestro, que dice: Para nosotros y nuestros hijos para siempre (Deuteronomio 29:28). Por eso renegaremos de él y lo mataremos si nos es posible. No tenemos que tomar en consideración sus milagros al igual que no debemos escuchar al profeta que dice que hay que cometer idolatría [...]. Moisés, nuestro maestro, ique en paz esté!, nos dijo: no practiquéis jamás la 
idolatría. Por eso conocemos que ese milagro que hace en nombre de la idolatría es sólo brujería y magia.” (Maimónides, 1987: 187).

La restauración del reino de Israel trae consigo un periodo de redención y retorno de los desterrados en diáspora en el que se reconstruirá el Templo y se restaurará la monarquía de los tiempos davídicos. Usando esta última figura el Rambam vuelve a una de las anunciaciones más antiguas del Tanaj hebreo sobre el mesías:

“ ¿Estoy viendo, pero no ahora, lo estoy mirando pero no está cercano en el tiempo: una estrella saldrá de Iaacov, un cetro surgirá de Israel, que dominará los límites de Moav y atravesará a todos los descendientes de Shet [hijo de Adán, metáfora de todas las naciones de la tierra]. Edom será una conquista, y Seír será una conquista de sus enemigos. E Israel triunfará. Y hará otra autoridad real en Iaacov que destruirá el remanente de la ciudad. [...] "iQuién puede sobrevivir a IHVH!" (Números 24:17-18).

De acuerdo con la lectura que realiza Maimónides de este texto, la profecía que se anuncia habría sido cumplida ya en la figura del primer mesías: David. No obstante, ella también da forma al segundo mesías, descendiente de la casa real de David y heredero de su trono. En ese sentido esto:

“Constituye uno de los signos fundamentales que caracterizan al mesías, especialmente, al pensar la relación entre el profetismo y lo político: para Maimónides el mesías debe ser una síntesis del conocimiento de la ley y el carisma del líder político caracterizado por la figura del rey” (Taub, 2012: $15)$. 
Siguiendo a Taub, esta consagración del mesías a la ley es una inversión de la profecía. Si la autoridad política de Moisés está construida sobre la base de la apertura del ciclo profético-legislativo, para el Mesías es lo político ya constituido de antemano por dicha ley, aquello que consagra su figura. En otras palabras, si lo político es inaugurado con la labor del profeta, ello se reanudada por la consagración que hace la ley de la figura del mesías (Taub, 2012). De allí que en la concepción de Maimónides, el mesías no modificará ni un ápice de la ley, ni asentara su autoridad en grandes prodigios y milagros. Su autoridad se desprenderá en su sumisión voluntaria a la ley que instauro el profeta:

“No creas que el Rey Mesías tendrá que realizar señales y prodigios, que traerá algo nuevo a la existencia, que revivirá a los muertos o cosas similares. No es así. Rabi Akiba fue un gran sabio, un maestro de la Mishná, sin embargo también fue el escudero de Bar Kojba. Él afirmó que éste era el Rey Mesías; él y todos los hombres sensatos de su generación compartieron esta creencia hasta que Bar Kojba fue asesinado por su injusticia, cuando se dieron cuenta que no era el Mesías. Y sin embargo los rabinos no le habían pedido una señal o un signo. El principio general es: esta Ley nuestra con sus estatutos y ordenanzas no puede cambiarse. Es para siempre y por toda la eternidad; no se le puede agregar o quitar” (Maimónodes, 1963: 239-

La tarea primordial del mesías respecto a la ley será hacer prevalecer a Israel en sus caminos, y traer de vuelta sus corazones a la Torá. Por esos en sus días, el cumplimiento y la observancia de los preceptos de Dios será el curso natural de la vida humana. Podemos afirmar de este modo, junto a Hartman que "la distinción entre el periodo mesiánico y el pre-mesiánico está en la naturaleza del dominio político" (Hartman, 1978: 10). Si el 
régimen que inaugura el profeta es el de la nación perfecta mediante la entrega de la ley, el gobierno que ejercerá el mesías no será una teocracia sino una nomocracia, un gobierno de la ley en la que Israel será restaurado.

Según Taub, el mesías está vinculado a tres tipos de expectativas: la personal, la nacional y la profética (Taub, 2012). Él, quien ocupará el trono de David y consagrará la ley, también impartirá justicia. En el mesías no solo se presentará la cuestión de la ley, sino también la de la justicia. Si el fundamento para que la ley fuese revelada fue el bienestar material y espiritual haciendo el bien de Dios y evitando el mal y la injusticia, la era que trae el mesías estará representada por el florecimiento de la rectitud entre los hombres en virtud de un vínculo directo con la ley de Dios (Taub, 2012). El Salmo 72:7 adelanta: “En sus días florecerán los justos y habrá abundancia de paz hasta que se consuma la luna eternamente".

Esta esperanza mesiánica, en la que el mundo conocerá finalmente al Dios vivo y verdadero, es descrita por Maimónides del siguiente modo: "En esa era no habrá hambruna ni guerra, tampoco envidia ni conflictos. Las bendiciones serán abundantes y el consuelo estará al alcance de todos. La única preocupación de todo el mundo será el conocimiento de IHVH” (Maimónodes, 1963: 242)

De cierto modo, la era mesiánica será para Maimónides una "pax judaica". Toda la humanidad se volverá sobre el Dios de Israel, aunque lo adorarán a su manera, respetando las 7 leyes de Noé. Se difundirá de este modo, la hegemonía moral del pueblo judío como nación con deberes de justicia excepcionales, pero al mismo tiempo como luz para otros pueblos.

Una aprensión que nos recuerda Strauss antes de terminar, "el Mesías es rey; eso quiere decir que su rango es inferior al del legislador-profeta. Mientras que el legislador profeta ha proclamado la ley divina, el rey obliga a los hombres a obedecer la ley" (Strauss, 2012: 242). Efectivamente, para Maimónides será una figura parecida a la de un profeta, pero nunca alcanzará la superioridad de Moisés. La primacía de la ley sobre la política, produce en el pensamiento de Maimónides una subyugación del Mesías al profeta legislador. 
Al concluir, es posible señalar que el mesianismo de Maimónides no es el fin del tiempo del hombre sino de la política. El problema de lo político que encuentra su inicio con Moisés en el Sinaí, halla su resolución en un rey que viene a dar un cumplimiento total a la Ley. De allí que "el mesianismo no sustituye las obligaciones legales del Sinaí, sino que retorna y realiza ese pacto en su totalidad" (Taub, 2012: 30). La era que se abre con la llegada del mesías no será un mundo nuevo ni tendrá una nueva Torá. Simplemente el pueblo judío retornara a la tierra que le fue prometida, desde donde los pueblos lo reconocerán y se vincularan con él legalmente, esto es, a través del conocimiento de la ley de Dios. Con esto culminará la historia como experiencia, pero no el tiempo. Ya no habrá mundo venidero, ni eras monoteístas. Viviremos conjuntamente en un eterno presente, que se nos presentará como intemporalidad. Cielo y tierra pasaran, más la ley prevalecerá.

\section{Bibliografía}

Davis, D. W. (1952). Torah in the Messianic Age and/or the Age to Come. Society of Biblical Litarature Philadelphia.

Hartman, D. (1978). Maimonides approach to messianism and its contemporary implications. A Journal of Jewish philosophy and Kabbalah.

Karmy, R. (2016). Escritos bárbaros: Ensayos sobre razón imperial y mundo árabe contemporáneo. Santiago de Chile: LOM.

Lerner, R. (1998). Historia de la filosofía política. En L. Strauss, \& J. Crospey, Moinsés Maimónides. Ciudad de Mexico: Fondo de Culutra Económica .

Maimónides. (1987). Sobre el Mesías. Carytas a los judíos de Yemen. Barcelona: Riopiedras.

Maimónides. (2005). Guia de Per'plejos. Madrid: Trotta.

Maimónodes. (1963). The Code of Maimonides (Mishneh Torah). Yale University Press. 
Strauss, L. (2009). La persecución y el arte de escribir. Buenos Aires: Amorrortu.

Strauss, L. (2012). El libro de Maimónides . Valencia: Pre-texto.

Taub, E. (2012). Ley, jusitcia y tiempo por venir: Maimónides y el ideal mesiánico. Abacronismo e Irrupción, 11-33.

Taub, E. (2014). El dilema de la filosofía judía: apuntes de Al-Farabi y Maimónides. En M. B. Taub, Atenas y Jerusalén . Buenos Aires: Prometeo Libros. 